\title{
PEA and luteolin synergistically reduce mast cell-mediated toxicity and elicit neuroprotection in cell-based models of brain ischemia
}

\author{
Edoardo Parrella ${ }^{a}$, Vanessa Porrini ${ }^{\mathrm{a}, *}$, Rosa Iorio ${ }^{\mathrm{a}}$, Marina Benarese ${ }^{\mathrm{a}}$, \\ Annamaria Lanzillotta ${ }^{\mathrm{a}}$, Mariana Mota ${ }^{\mathrm{a}}$, Mariella Fusco ${ }^{\mathrm{c}}$, Paolo Tonin ${ }^{\mathrm{b}}$, \\ PierFranco Spano ${ }^{\mathrm{a}, \mathrm{b}}$, Marina Pizzi ${ }^{\mathrm{a}, \mathrm{b}}$ \\ a Division of Pharmacology, Department of Molecular and Translational Medicine, National Institute of Neuroscience, University of Brescia, Italy \\ b IRCCS San Camillo, Venezia, Italy \\ ${ }^{\mathrm{c}}$ Epitech Group s.r.l., Saccolongo, Padova, Italy
}

\section{A R T I C L E I N F O}

\section{Article history:}

Received 2 March 2016

Received in revised form 8 July 2016

Accepted 12 July 2016

Available online 14 July 2016

\section{Keywords:}

PEA

Luteolin

Mast cells

Neurons

Brain ischemia

Neuroinflammation

\begin{abstract}
A B S T R A C T
The combination of palmitoylethanolamide (PEA), an endogenous fatty acid amide belonging to the family of the $\mathrm{N}$-acylethanolamines, and the flavonoid luteolin has been found to exert neuroprotective activities in a variety of mouse models of neurological disorders, including brain ischemia. Indirect findings suggest that the two molecules can reduce the activation of mastocytes in brain ischemia, thus modulating crucial cells that trigger the inflammatory cascade. Though, no evidence exists about a direct effect of PEA and luteolin on mast cells in experimental models of brain ischemia, either used separately or in combination. In order to fill this gap, we developed a novel cell-based model of severe brain ischemia consisting of primary mouse cortical neurons and cloned mast cells derived from mouse fetal liver (MC/9 cells) subjected to oxygen and glucose deprivation (OGD).

OGD exposure promoted both mast cell degranulation and the release of lactate dehydrogenase (LDH) in a time-dependent fashion. MC/9 cells exacerbated neuronal damage in neuron-mast cells co-cultures exposed to OGD. Likewise, the conditioned medium derived from OGD-exposed MC/9 cells induced significant neurotoxicity in control primary neurons. PEA and luteolin pre-treatment synergistically prevented the OGD-induced degranulation of mast cells and reduced the neurotoxic potential of MC/9 cells conditioned medium. Finally, the association of the two drugs promoted a direct synergistic neuroprotection even in pure cortical neurons exposed to OGD.

In summary, our results indicate that mast cells release neurotoxic factors upon OGD-induced activation. The association PEA-luteolin actively reduces mast cell-mediated neurotoxicity as well as pure neurons susceptibility to OGD.
\end{abstract}

(c) 2016 Elsevier B.V. All rights reserved.
Abbreviations: AD, Alzheimer's Disease; BBB, brain blood barrier; CM, conditioned medium; CNS, central nervous system; DIV, days in vitro; DMSO, dimethyl sulfoxide; LDH, lactate dehydrogenase; MCs, mast cells; NAEs, N-acylethanolamines; NF-kB, Nuclear factor-kB; OGD, oxygen and glucose deprivation; PD, Parkinson's Disease; PEA, palmitoylethanolamide; PPARs, Peroxisome proliferator-activated receptors; SEM, standard error of the mean; TBI, traumatic brain injury

* Correspondence to: Division of Pharmacology, Department of Molecular and Translational Medicine, National Institute of Neuroscience, University of Brescia, Viale Europa 11, 25123 Brescia, Italy.

E-mail addresses: edoardo.parrella@unibs.it (E. Parrella), v.porrini@unibs.it (V. Porrini), rosa91i@hotmail.it (R. Iorio), marina.benarese@unibs.it (M. Benarese), annamaria.lanzillotta@unibs.it (A. Lanzillotta), m.coelhodamota@unibs.it (M. Mota), mariella.fusco@epitech.it (M. Fusco), paolo.tonin@ospedalesancamillo.net (P. Tonin), pierfranco.spano@unibs.it (P. Spano), marina.pizzi@unibs.it (M. Pizzi).

\section{Introduction}

Mast cells (MCs) are bone marrow-derived perivascular resident cells widely distributed throughout virtually all vascularized tissues where they normally reside in proximity to blood vessels, nerves, smooth muscle cells, mucus-producing glands, and hair follicles. MCs can be especially numerous in anatomical regions that are directly exposed to the environment such as the skin, airways, and gastrointestinal tract (Metz et al., 2007). Because of their peculiar anatomical distribution, MCs act as first line of defense in the immune system against invading pathogens and environmental antigens and allergens. Initially known mainly for their pathogenic role in allergic and anaphylactic reactions, it is now well documented MCs involvement in processes of innate and adaptive immunity and inflammation. Mastocytic activation 
occurs upon response to a wide range of physical and chemical stimuli, including trauma, hypoxia, allergens, toxic substances, complement factors, endogenous and exogenous peptides, cytokines and other inflammatory mediators (Galli et al., 2005). MCs also are present in the central nervous system (CNS) and they have been identified in different mammalian brain regions, including meninges, choroid plexus, olfactory bulb, mesencephalon, parenchima of thalamus and hypothalamus, hippocampus and enthorinal cortex (Dropp, 1979; Silver and Curley, 2013; Theoharides, 1990). MCs have been associated with various neuroinflammatory conditions of the CNS, such as stroke, multiple sclerosis, traumatic brain injury and eventually Alzheimer's Disease (AD) and Parkinson's Disease (PD) (Nelissen et al., 2013). A body of evidence points out the involvement of MCs in the pathophysiology of brain ischemia (Lindsberg et al., 2010; Strbian et al., 2009). After ischemia, MCs translocate from brain perivascular positions and can be more abundantly found in the ischemic cerebral area where they release their granules contents and mediators of inflammation (Caltagirone et al., 2015; Hu et al., 2004; Lindsberg et al., 2010; Silverman et al., 2000; Strbian et al., 2006). In rodent models of brain ischemia, both genetic deficiency or pharmacological inhibition of MCs led to a significant reduction of ischemic damages, including brain blood barrier (BBB) disruption, cerebral edema, and neutrophil infiltration (Mattila et al., 2011; Strbian et al., 2006).

In the light of the above observations, MCs appear as an attractive target for the treatment of brain ischemia. In this study we tested PEA (palmitoylethanolamide) and luteolin (3', 4', 5, 7-tetrahydroxyflavone), two natural compounds potentially active as MCs modulators, on cell-based models of brain ischemia.

PEA is an endogenous lipid amide belonging to the family of the $\mathrm{N}$-acylethanolamines (NAEs). PEA can be found in mammalian tissues, especially the brain. It has been proposed that PEA may be produced "on demand" in the CNS during brain injury to counteract neuronal damage (Esposito et al., 2014; Hansen, 2010). PEA is endowed with anti-inflammatory and analgesic properties and it has been investigated in both cellular and animal models of chronic pain (De Filippis et al., 2011; LoVerme et al., 2006), AD (D'Agostino et al., 2012; Scuderi et al., 2014; Scuderi et al., 2012), PD (Esposito et al., 2012), uveitis (Impellizzeri et al., 2015), diabetic retinopathy (Paterniti et al., 2015) and stroke (Ahmad et al., 2012; Garg et al., 2010).

Luteolin is a flavonoid present in many plants, including various fruits, vegetables and medicinal herbs (Lopez-Lazaro, 2009). Luteolin and its congeners exhibit anti-inflammatory, antioxidant, neuroprotective and anti-carcinogenic activities (Dajas et al., 2003; Guerra-Araiza et al., 2013) and have been reported to have a potential for the treatment of autism (Theoharides et al., 2013), multiple sclerosis (Theoharides, 2009), cancer (Attoub et al., 2011) and stroke (Qiao et al., 2012; Qiao et al., 2014; Zhao et al., 2011).

Several reports indicate that the two molecules used separately can reduce MCs activation in a variety of in vitro and in vivo experimental models (De Filippis et al., 2011; De Filippis et al., 2013; Esposito et al., 2011; Facci et al., 1995; Jeon et al., 2014; Jin et al., 2011; Kritas et al., 2013; Skaper et al., 2013, 1996a, 1996b).

Moreover, recent findings indicate that the combination PEAluteolin is effective in reducing inflammation in animal models of spinal cord injury (Paterniti et al., 2013), AD (Paterniti et al., 2014), traumatic brain injury (TBI) (Cordaro et al., 2015), anxiety/depression (Crupi et al., 2013), arthritis (Impellizzeri et al., 2013), PD (Siracusa et al., 2015) and brain ischemia (Caltagirone et al., 2015), and in promoting the maturation of oligodendrocytes precursor cells in vitro (Barbierato et al., 2015). Finally, it has been recently shown that the combination PEA-luteolin is able to reduce the ischemia-induced MCs infiltration in a rat model of brain ischemia (Caltagirone et al., 2015).
The aim of this study was to test the efficacy of the combination PEA-luteolin in reducing neuronal damage in cell-based models of brain ischemia, with particular focus on the effect of the two compounds on MCs-mediated neurotoxicity.

\section{Results}

\subsection{OGD promotes $M C / 9$ cells activation}

We tested the effect of OGD on degranulation and cellular damage of MC/9 cells. Cultured MC/9 cells were exposed to 3, 6, 8, or $16 \mathrm{~h}$ of OGD, then harvested and processed. As pointed out by previous studies on primary rat MCs, OGD induced mastocytic activation (Hu et al., 2005). When stained with toluidine blue and observed by light microscopy, most MC/9 cells in the control group showed metachromatic granules and membrane integrity (Fig. 1A). OGD exposure resulted in the appearance of numerous MC/9 cells undergoing degranulation (Fig. 1A). Granule release in MC/9 cells subjected to OGD was significantly higher than control group after $6 \mathrm{~h}$ of anoxic insult, and increased further thereafter (Fig. 1B). $\beta$ hexosaminidase release, another marker of mastocytic degranulation, increased similarly in a fashion dependent from OGD duration, becoming significantly different than control after $6 \mathrm{~h}$ of OGD exposure (Fig. 1C). Together with the degranulation, OGD promoted also LDH release, a marker of cellular damage. A significant increase was observed after $6 \mathrm{~h}$ of anoxic insult, followed by a progressive growth over time (Fig. 1D).

\subsection{OGD-activated MC/9 cells release neurotoxic factors}

Next, we investigated whether MC/9 cells could modulate neuronal damage induced by OGD. Based on our results showing a significant mastocytic activation after $6 \mathrm{~h}$ of OGD, in the next experiments we challenged MC/9 cells with $6 \mathrm{~h}$ of anoxic condition. In a first experimental approach, we established a cell model of cerebral ischemia based on a trans-well co-culture system, with neurons aderent in the well and MC/9 cells seeded in the upper trans-well (Fig. 2A). This system allowed us to model the interactions between neurons and MCs in pathophysiological conditions of cerebral ischemia. Pure neurons or neurons- MC/9 cells cocultures were subjected to $6 \mathrm{~h}$ of OGD, followed by $24 \mathrm{~h}$ of reperfusion, in accordance with the OGD experimental procedure we developed for neuronal cultures (Lanzillotta et al., 2013, 2010; Sarnico et al., 2009). Neurons- MC/9 cells co-cultures displayed higher cell death when compared to pure neurons (Fig. 2B). This effect is likely due to MC/9 cells-mediated amplification of the neurotoxic response rather than $\mathrm{MC} / 9$ cells release of $\mathrm{LDH}$. The LDH release in sister cultures of MC/9 cells, seeded at the same density as in the co-cultures, revealed negligible enzyme discharge in the medium when compared to the LDH released by pure neuronal cultures (Fig. 2C).

In order to further investigate the adverse effect of MCs on neurotoxicity, using a second experimental approach, conditioned medium (CM) from OGD-activated MC/9 cells was added to separate pure neurons. CM from MC/9 cells subjected to $6 \mathrm{~h}$ of OGD was collected at the end of the anoxic event, or after a further reperfusion period, and transferred to neuronal cultures (Fig. 2D). The addition of CM harvested from MC/9 cells immediately after OGD did not induce significant toxicity in neurons (Fig. 2E). Conversely, the addition of $\mathrm{CM}$ collected from MC/9 cells exposed to OGD followed by $24 \mathrm{~h}$-reperfusion extremely exacerbated the cell death (Fig. 2E). This result suggests that the release of neurotoxic factors from MC/9 cells is responsible for the enhanced neuronal cell death produced by $\mathrm{MC} / 9$ cells during co-exposure to OGD. 
a
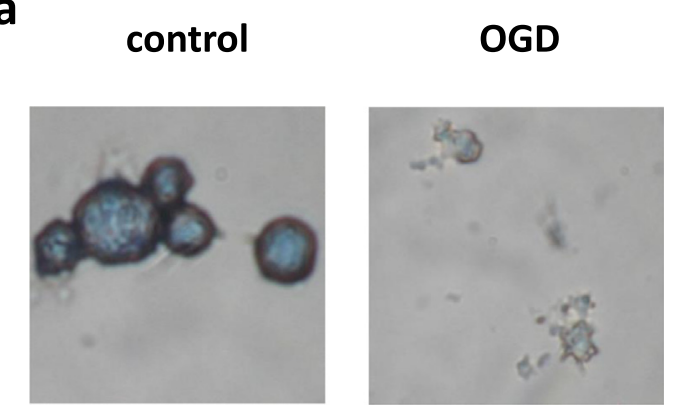

C

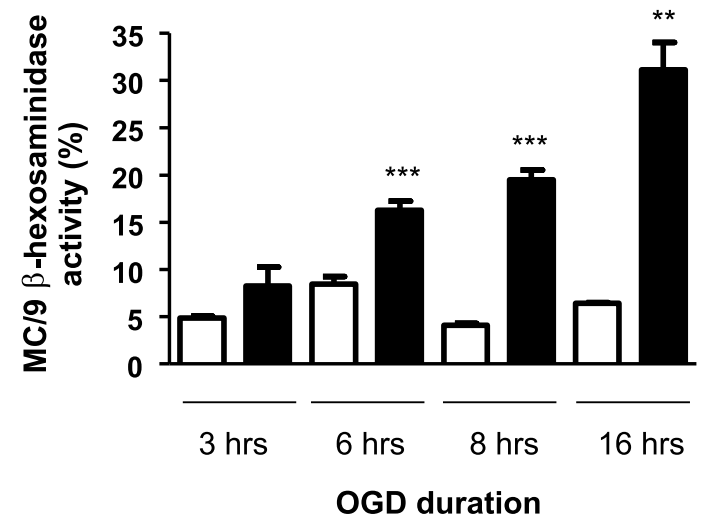

b

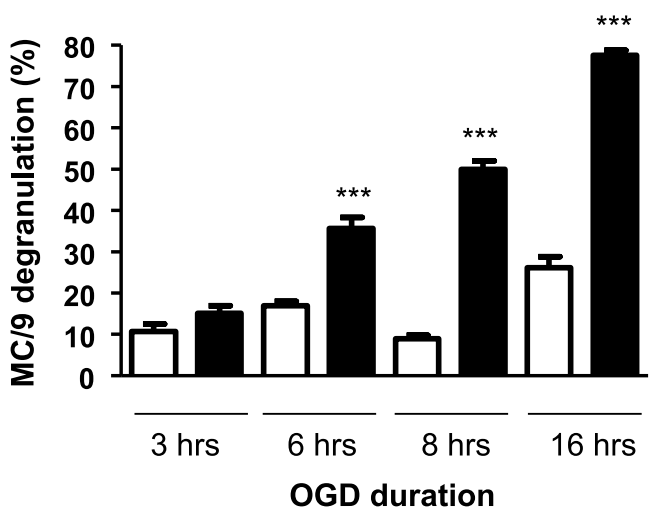

d

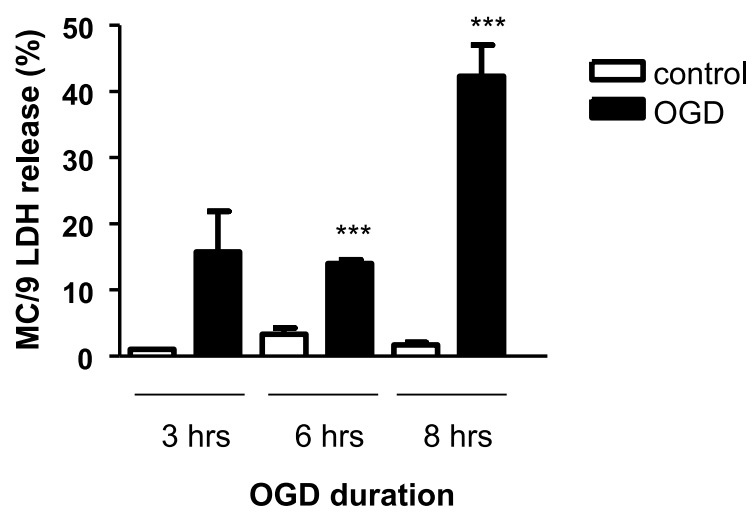

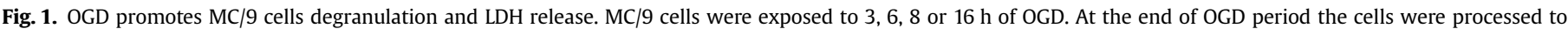

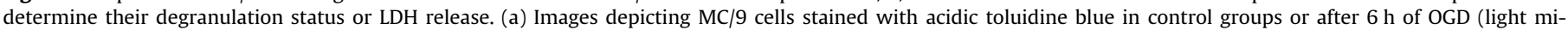

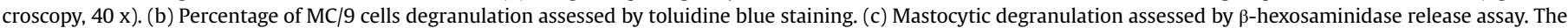

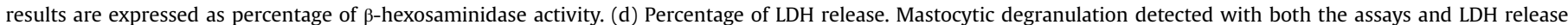

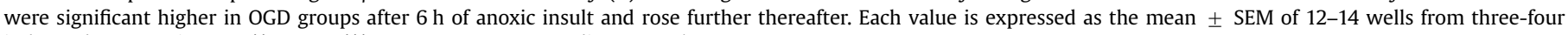
independent experiments. ${ }^{* *} p<0.01,{ }^{* * *} p<0.001 v s$ corresponding control, $t$-test.

\subsection{Pre-treatment with PEA and luteolin synergistically reduces OGD-dependent activation of MC/9 cells}

We evaluated whether PEA and luteolin, tested at concentration ranges $1 \mathrm{nM}$ to $100 \mu \mathrm{M}$, could dampen mastocytic activation upon OGD treatment. The drugs were added to cell cultures $15 \mathrm{~h}$ before OGD and maintained in the medium during the $6 \mathrm{~h}$ of anoxic event. Mastocytic degranulation was assessed at the end of OGD period. PEA did not affect degranulation of MC/9 cells exposed to OGD (Fig. 3A). Pre-treatment of MC/9 cells with luteolin significantly prevented mastocytic degranulation at the concentration range 10-100 nM (Fig. 3B). When the two drugs were added together at ineffective concentrations per se, PEA 10$100 \mathrm{nM}$ and luteolin $1 \mathrm{nM}$, they considerably decreased MC/9 cells degranulation, suggesting a synergistic modulatory activity on stimulated MCs (Fig. 3C). In line with the lower degranulation observed, the PEA-luteolin combination significantly reduced the LDH release from activated $\mathrm{MC} / 9$ cells, a marker of cellular damage (Fig. 3D).

\subsection{The combination of PEA and luteolin reduces neurotoxicity mediated by OGD-activated MC/9 cells}

Next we determined if the synergistic effect exerted by PEA and luteolin on MC/9 cells activation could limit the neurotoxic potential of their CM. The drug association, luteolin $1 \mathrm{nM}$ with PEA $100 \mathrm{nM}$, was added to the MC/9 cells $15 \mathrm{~h}$ before OGD exposure and maintained till $24 \mathrm{~h}$ after the OGD. The CM was then harvested and transferred to neurons. The relative LDH release in control cells and in cells incubated with CM was $0.06 \pm 0.01$ and $0.36 \pm 0.1$ respectively (mean \pm SEM). The combination of PEA and luteolin significantly reduced the neurotoxic activity of $\mathrm{CM}$ derived from MC/9 cells exposed to OGD (Fig. 4).

\subsection{The combination of PEA and luteolin promotes synergistic neu- roprotection in neurons exposed to OGD}

To investigate whether PEA and luteolin were also endowed with direct neuroprotective property, different concentrations of the two molecules, alone or in association, were tested on neurons previously exposed to $3 \mathrm{~h}$ of OGD. The drugs were added to the cell cultures after the anoxic event and maintained in the recovery medium for the following $24 \mathrm{~h}$. In line with previous data (Lanzillotta et al., 2013, 2010; Sarnico et al., 2009), the relative LDH release in control neurons and in neurons subjected to $3 \mathrm{~h}$ of OGD was $0.1 \pm 0.02$ and $0.32 \pm 0.02$ respectively (mean \pm SEM). PEA did not exhibit significant neuroprotective effect at any concentration tested in the range $10 \mathrm{pM}-5 \mu \mathrm{M}$ (Fig. 5A). Luteolin showed a significant neuroprotective activity at concentration ranging from $100 \mathrm{nM}$ to $1 \mu \mathrm{M}$, whereas it lost its effect at higher concentrations (Fig. 5B). As observed in MC/9 cells, when the drugs were administered together at per se ineffective or weakly active concentrations, PEA $10-100 \mathrm{nM}$ and luteolin $1 \mathrm{nM}$, they significantly reduced the neuronal cell death. This result suggests 
a

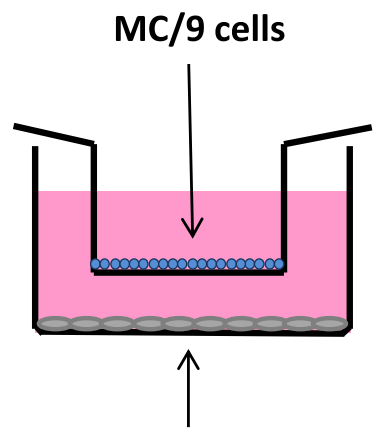

neurons b

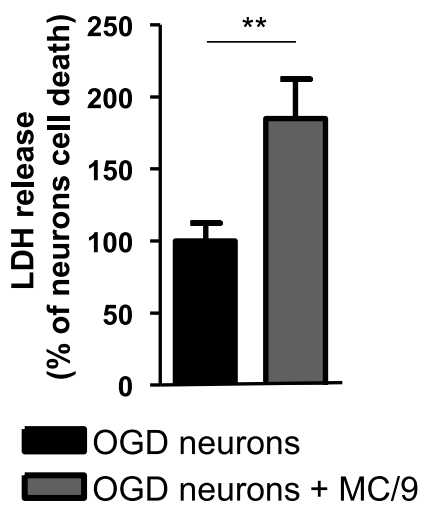

C

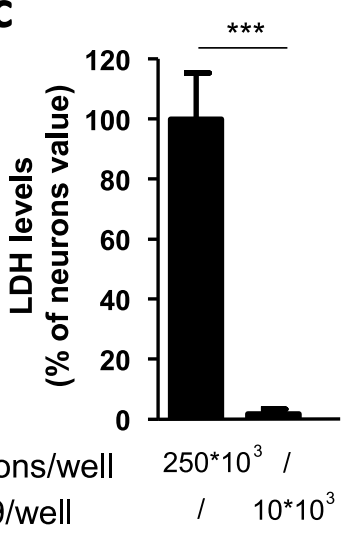

d
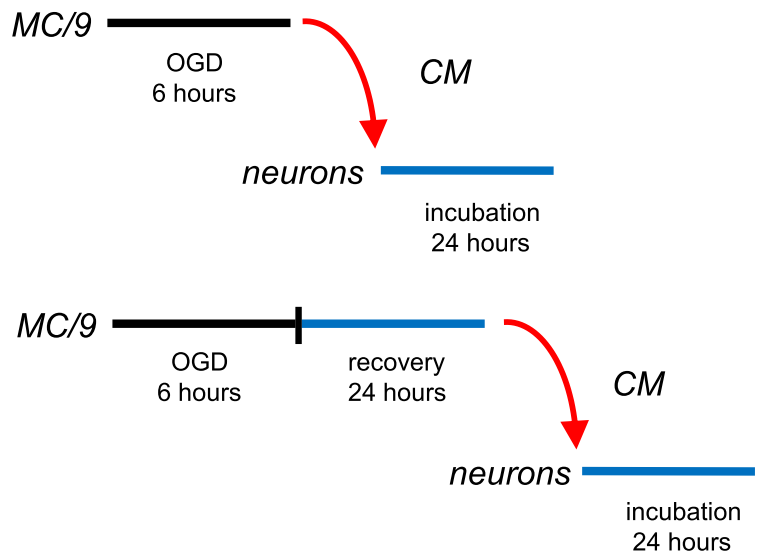

e
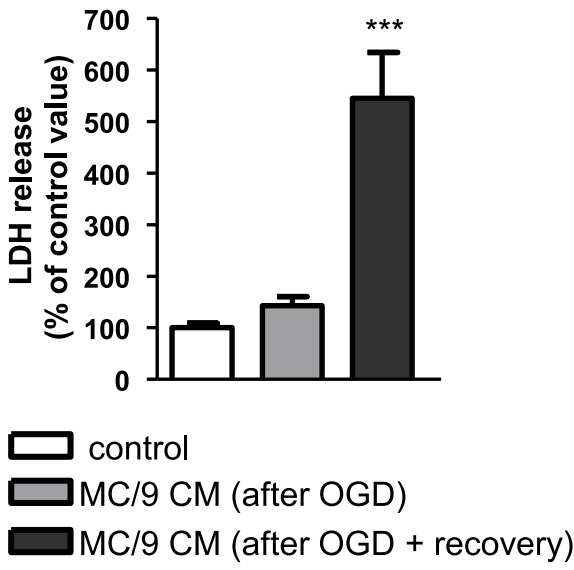

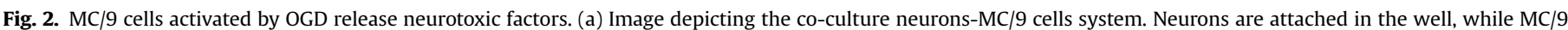

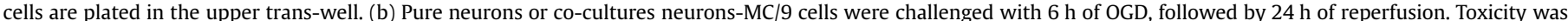

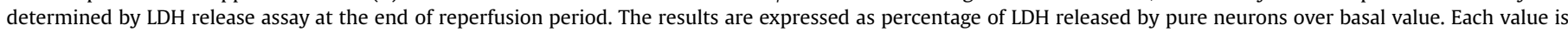

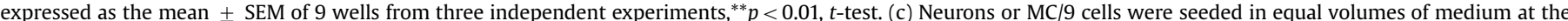

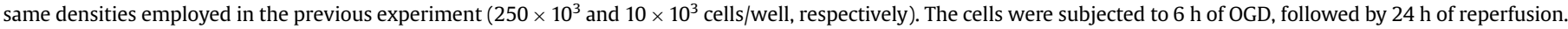

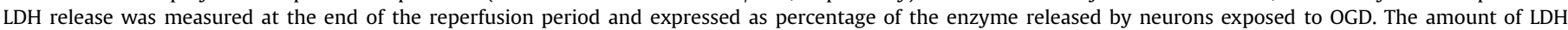

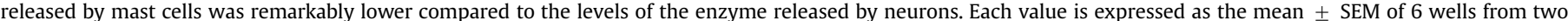

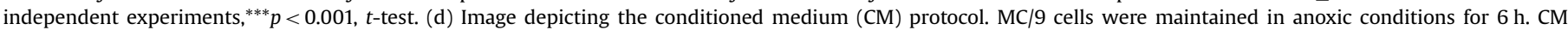

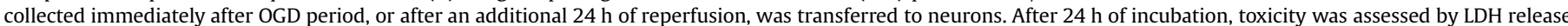

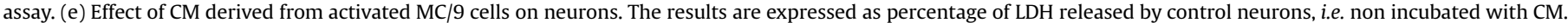

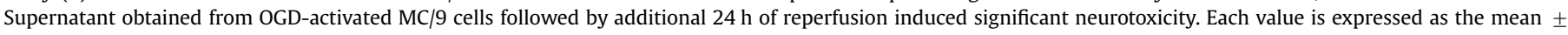
SEM of 9 wells from three independent experiments, ${ }^{* * *} p<0.001$, one-way ANOVA followed by Dunnet's multiple comparison test.

that PEA and luteolin synergistic association is also endowed with direct neuroprotective activity (Fig. 5C).

\section{Discussion}

Our results show that OGD exposure induces degranulation of the mastocytic MC/9 cells line. In accordance with previous findings, the amplitude of MCs degranulation is dependent on the duration of anoxic insult (Hu et al., 2005). Degranulation was accompanied by significant leakage of LDH, indicating a cytotoxic effect induced by OGD. Active exocytic secretion and cytotoxic release of cellular content are two types of mastocytic degranulation that can differently occur in response to specific exogenous stimuli or can be tuned by the stimulus intensity. For instance, either linoleic or arachidonic acids can induce mastocytic degranulation, although only linoleic acid induces a concomitant LDH release (Masini et al., 1990). Eosinophil peroxidase at low doses induces mastocytic degranulation, but at higher doses it promotes both cytotoxic degranulation and LDH release (Henderson et al., 1980). Our experimental setting allowed to model the injury produced by a severe brain ischemia on MCs resulting in their cell damage and degranulation.

In neuron-MC/9 co-cultures subjected to OGD, the presence of MCs exacerbated the neuronal cell death. A similar neurotoxic effect was found when neurons were treated with supernatants derived from MC/9 cells previously exposed to OGD. Worth of note, only the CM collected after a recovery period following OGD, and not the supernatant obtained immediately after OGD, was able to induce massive neurotoxicity. A body of evidence shows that MCs activation occurs in two phases (Silver and Curley, 2013; Theoharides, 1990). The first and rapid response occurs within seconds after mastocytic activation and consists in the degranulation of MCs, i.e. the release of pre-formed mediators stored in MCs granules. Granules contents include histamine, heparin, serotonin, proteases, proteoglycans, cathepsin $G$ and cytokines (Nelissen et al., 2013; Silver and Curley, 2013). The second and slow phase is the release of newly synthetized cytokines and 
a

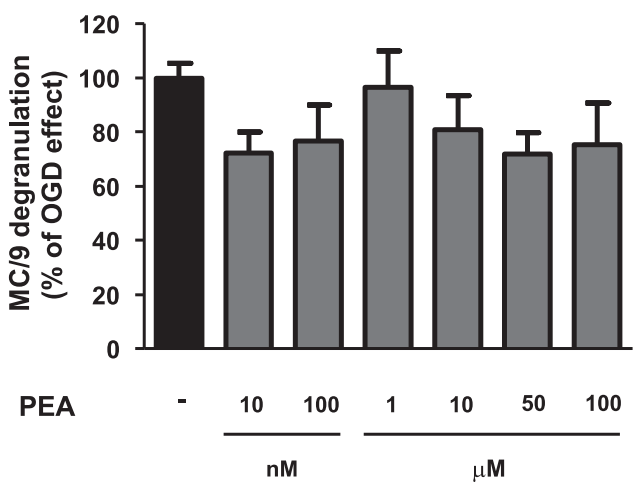

C

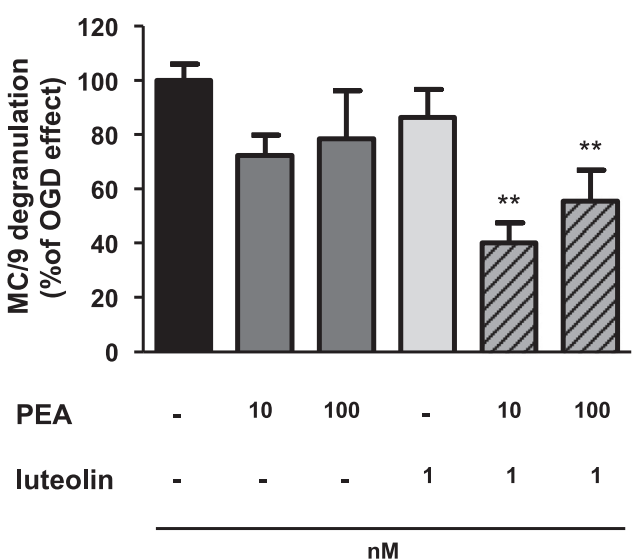

b

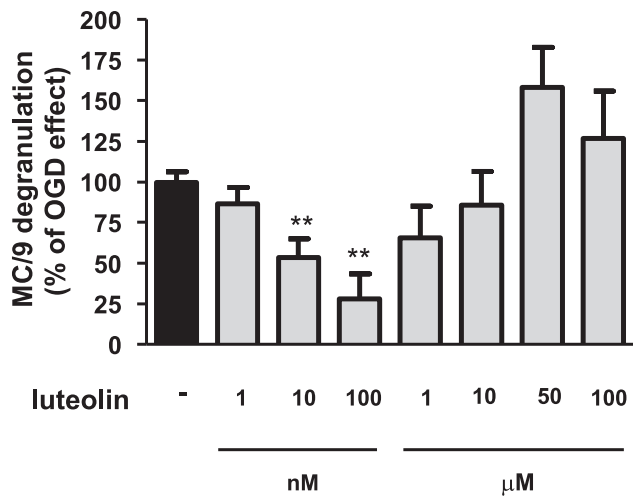

d

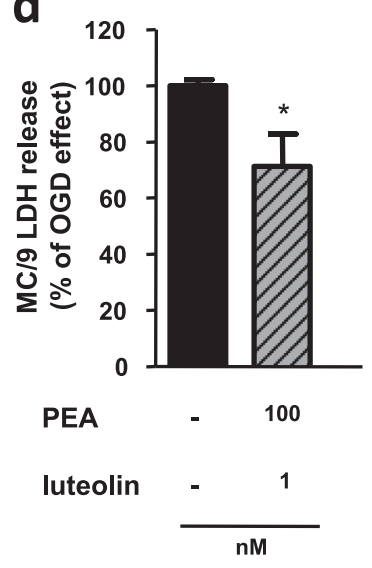

OGD + vehicle

$\square O G D+P E A$

$\square O G D+$ luteolin

EZT

OGD + PEA + luteolin

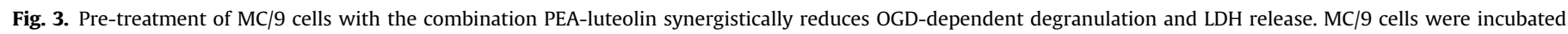

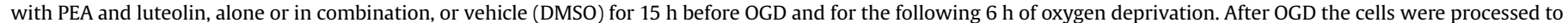

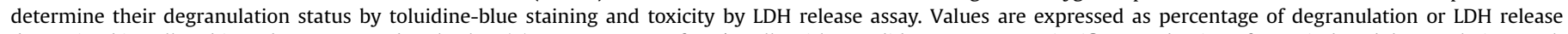

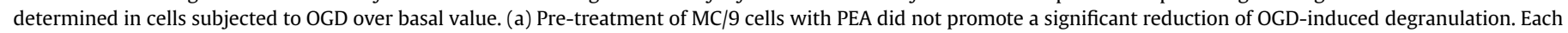

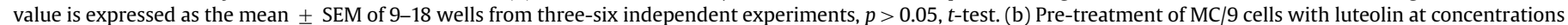

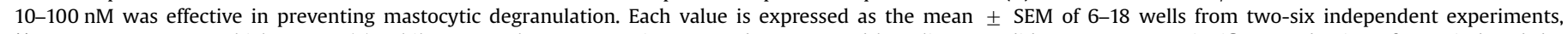

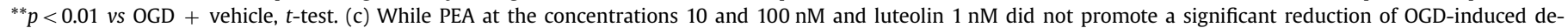

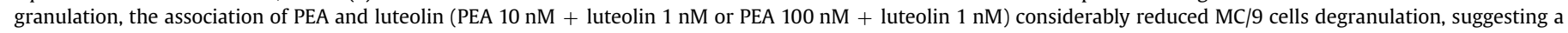

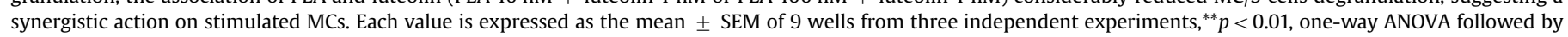

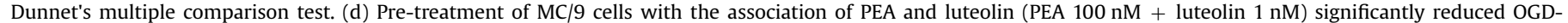
induced toxicity. Each value is expressed as the mean \pm SEM of 9 wells from three independent experiments, ${ }^{*} p<0.005, t$-test.

chemokines and occurs within several hours after mastocytic activation (Nelissen et al., 2013; Silver and Curley, 2013). The molecules released in the different phases of mastocytic activation can promote and modulate the organism reaction to exogenous stimuli, inducing inflammatory processes such as the recruitment and the activation of inflammatory cells, vasodilation and plasma extravasation, but can also drive anti-inflammatory or immunosuppressive responses (Christy and Brown, 2007; Galli et al., 2005; Marshall, 2004; Metz et al., 2007; Silver and Curley, 2013). Our results suggest that under severe ischemia MCs can release neurotoxic factors that are mainly synthetized ex novo in the postanoxia period.

Our experiments demonstrate that PEA per se was inactive in reducing either $\mathrm{MC} / 9$ cells activation or neuronal cell death when the cell cultures were exposed to OGD. By contrast, luteolin was able to decrease mastocytic activation at the concentration range $10-100 \mathrm{nM}$ and neuronal cell death at the $100 \mathrm{nM}-1 \mu \mathrm{M}$ range. The effects promoted by luteolin on MCs and neurons disappeared at higher concentrations. Multiple factors could account for this phenomenon, including a possible pro-oxidant activity of the molecule at higher doses (Lin et al., 2008). Future studies are needed to determine the mechanism behind the loss of neuroprotective activity of luteolin.

The combination of the molecules PEA: luteolin 10:1 or 100:1 at the nanomolar range maximally reduced the degranulation and LDH release from MCs and induced neuroprotection in nearly pure cortical neurons exposed to OGD. Notably, in primary neurons the drug combination was effective when added after the OGD exposure. Either in neurons or in MCs the effective combination was obtained using the compounds at concentrations per se inactive. This evidence strongly suggests the synergistic activity of the two drugs in producing an overall neuroprotection in models of brain ischemia. The synergistic action of PEA and luteolin is relevant also in the light of the severe anoxic conditions employed in this study, that can reasonably mimic the environment encountered by MCs in the core region of the ischemic brain.

The mechanism by which PEA-luteolin association can promote neuroprotection in MCs/neurons system subjected to anoxic insult remains to be explored yet. Previous studies have shown that PEAluteolin combination can reduce inflammatory processes associated with spinal cord injury and TBI by restoring basal expressions of peroxisome proliferator-activated receptors (PPARs) and by limiting nuclear factor-kB (NF-kB) activation (Cordaro et al., 2015; Paterniti et al., 2013). Moreover, PEA-luteolin association 

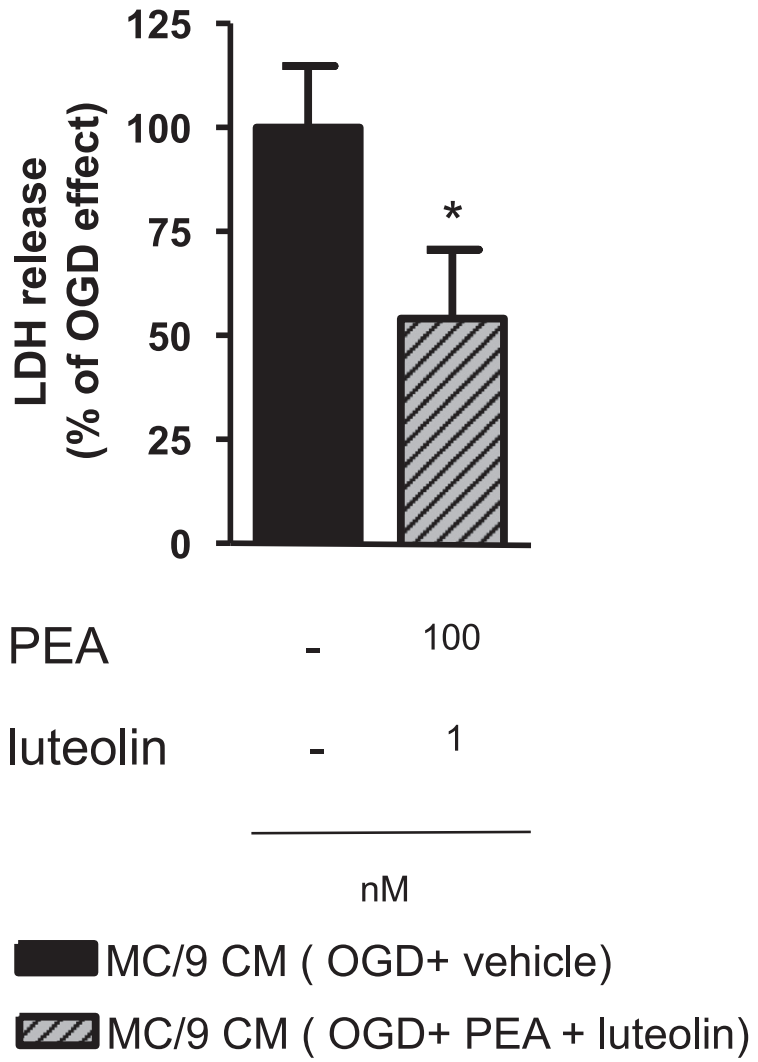

Fig. 4. The combination PEA-luteolin reduces the neurotoxicity mediated by OGDactivated MC/9 cells. MC/9 cells were treated with the vehicle or the association of PEA and luteolin (PEA $100 \mathrm{nM}$ + luteolin $1 \mathrm{nM}$ ) for $15 \mathrm{~h}$ before OGD. The incubation with the vehicle (DMSO) or the drugs was carried on for the $6 \mathrm{~h}$ of OGD and the following $24 \mathrm{~h}$ of reperfusion. CM obtained as previously described was used to incubate cortical neurons for $24 \mathrm{~h}$, at the end of which the cellular damage was assessed by $\mathrm{LDH}$ release assay. Results are expressed as percentage of $\mathrm{LDH}$ released by neurons incubated with $\mathrm{CM}$ from $\mathrm{MC} / 9$ cells subjected to OGD over basal value. The combination PEA-luteolin significantly reduced the neurotoxicity mediated by OGD-activated MCs. Each value is expressed as the mean \pm SEM of 6 wells from two independent experiments, ${ }^{*} p<0.05$, $t$-test.

was demonstrated to reduce TNF- $\alpha$ and IL- $1 \beta$ levels in animal models of TBI and arthritis (Cordaro et al., 2015; Impellizzeri et al., 2013). Notably, both TNF- $\alpha$ and IL-1 $\beta$, known to induce direct neurotoxicity, can be released by activated MCs (Galli et al., 2005; Silver and Curley, 2013). These classical factors released during neurotoxic activation of MCs, and possibly other yet unidentified molecules, deserve further investigation.

A number of clinical studies confirmed the anti-inflammatory and analgesic properties of PEA, while basically no adverse effects were observed at pharmacological doses (Skaper et al., 2014). Few clinical studies exist for luteolin, but none of them reported major side effects (Taliou et al., 2013; Theoharides et al., 2012). Importantly, PEA and luteolin are natural products present in many foods. PEA is a food component of egg yolk, peanut oil and soybean lecithin (Esposito and Cuzzocrea, 2013). Consumption of these foods has been associated to anti-allergic and anti-inflammatory activities in laboratory animals and to a decrease of rheumatic fever in children, likely via PEA activity (Esposito and Cuzzocrea, 2013). Luteolin and its glycosides are widely distributed in the plant kingdom and have been identified in many edible plants (Lopez-Lazaro, 2009) (for a complete list, see "USDA database for the flavonoid content of selected foods"). Different studies indicate that luteolin can be absorbed after oral administration and that its bioavailability and metabolism are sufficient to allow it to exert its biological activities in vivo (Lopez-Lazaro, 2009).
The effect promoted by the pre-treatment with PEA-luteolin on OGD-activated MCs would suggest a possible clinical application of the drug combination in the prevention of stroke, for instance, in association with antithrombotic and antihypertensive agents. In this study we did not explore the effects of the drugs when added to MCs just after the OGD exposure, though we showed that MCs can release neurotoxic factors synthetized ex novo in the post-OGD period and that PEA-luteolin can limit the cell death when added to cultured neurons after the OGD. These findings, together with the evidence that PEA-luteolin are neuroprotective when administered to rats after the onset of brain ischemia (Caltagirone et al., 2015), raise the possibility that the drug combination may be of therapeutical value even when administered in the acute post ischemic phase.

\section{Conclusions}

Here we present a novel cell-based model to study the role of MCs in brain ischemia. The use of MC/9 cells and the co-culture system neurons-MC/9 cells under OGD conditions allowed us to analyze the response of MCs to ischemic stimuli and to evaluate the contribution of MCs in anoxia-induced neurotoxicity. Moreover, we demonstrated that the combination of the two natural compounds, PEA and luteolin, at very low concentrations, elicits a synergistic modulation of MCs-mediated neurotoxicity beside a direct neuroprotection of primary neurons. Future studies are needed to further elucidate the mechanism underlying the synergy of the PEA-luteolin association.

\section{Experimental procedures}

\subsection{Drugs}

PEA and luteolin were provided by Epitech Group s.r.l., Saccolongo, Italy. The compounds were dissolved in dimethyl sulfoxide (DMSO, Sigma-Aldrich, Saint Louis, MO, U. S. A.). DMSO was used at a final dilution lower than $1 \%$, a concentration that we found having no effect on MCs and neurons in our experimental conditions (data not shown).

\subsection{Cell cultures}

\subsubsection{Primary cultures of mouse cortical neurons}

Primary cultures of mouse cortical neurons derived from C57BL/6 mice (Charles River Italia, Calco, Italy). All animal studies were approved by the Animal Research Committees of the University of Brescia and follow the Directive 2010/63/EU of the European Parliament and of the Council of 22 September 2010 on the protection of animals used for scientific purposes. The neurons were obtained from cortices of 15-day embryonic mice as previously described (Lanzillotta et al., 2013). The cells were seeded at the density $2.5 \times 10^{5}$ cells/well in 24-well plates (Nunc-Thermo Scientific, Waltham, MA, U.S.A.) coated with $10 \mu \mathrm{g} / \mathrm{mL}$ poly-1-lysine (Sigma-Aldrich, Saint Louis, MO, U.S.A.) using Neurobasal medium (Invitrogen Corporation, Carlsbad, CA, USA) supplemented with 2\% B27 (Invitrogen Corporation), $0.5 \mathrm{mM}$ l-glutamine (Euroclone, Celbio spa, Milan, Italy) and $50 \mathrm{U} / \mathrm{mL}$ penicillin/streptomicin (Euroclone). The neurons were used after 11-12 days in vitro (DIV).

\subsubsection{MC/9 cells}

MC/9 cells are cloned MCs derived from mouse fetal liver (ATCC, Rockville, MD, U. S. A.). The cell line was cultured in appropriate growth medium (High glucose Dulbecco's Modified 
a

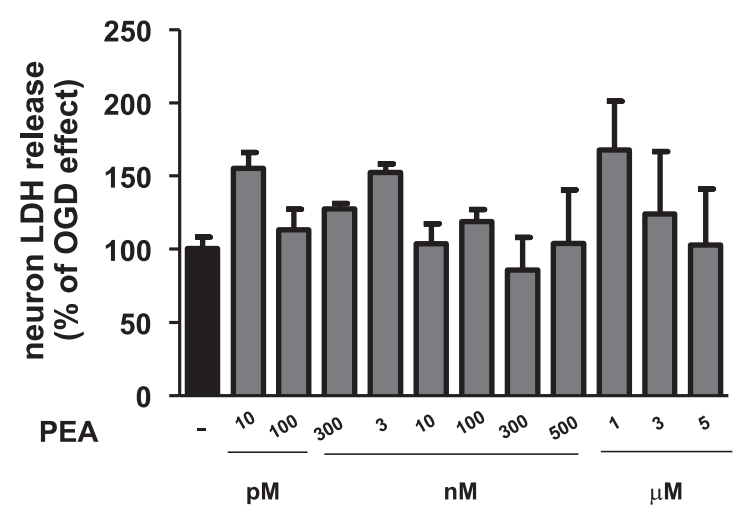

C

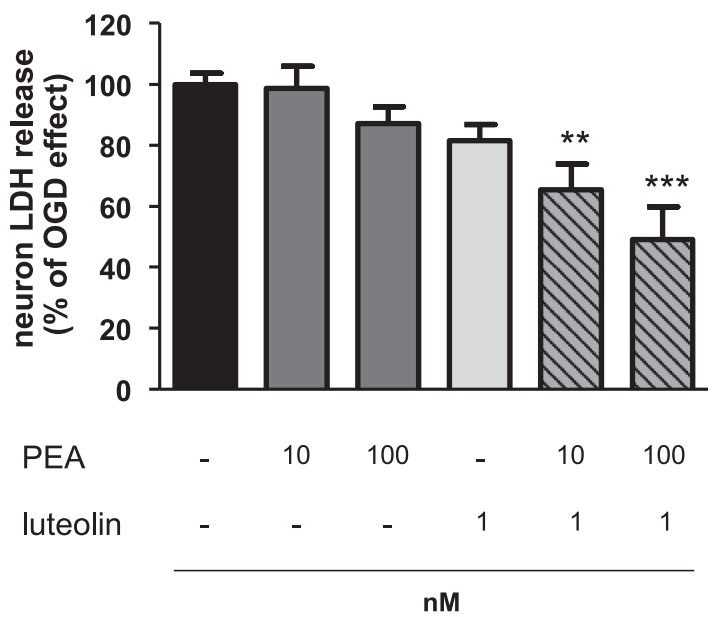

$\mathrm{nM}$ b

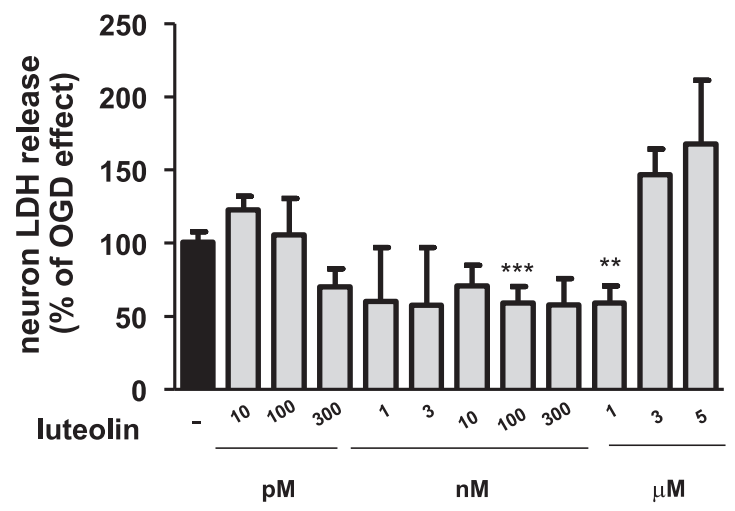

$\square$ OGD + vehicle
$\square$ OGD + PEA
$\square$ OGD + luteolin
$\square$ OGD + PEA + luteolin

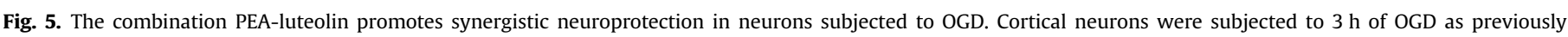

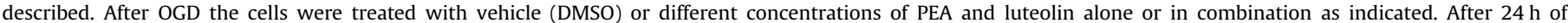

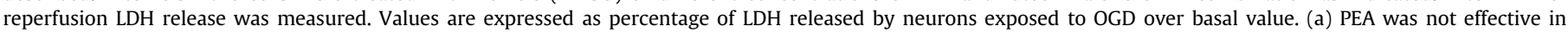

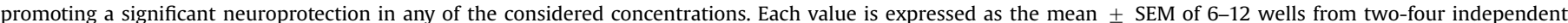

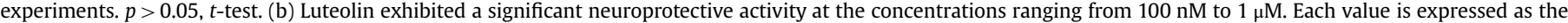

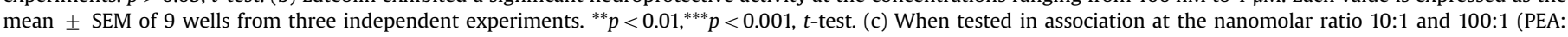

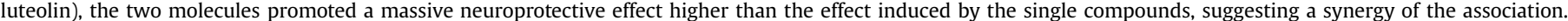

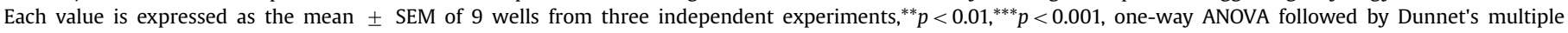
comparison test.

Eagle's Medium - Euroclone - supplemented with $2 \mathrm{mM}$ L-glutamine and 10\% fetal bovine serum -Euroclone-, $0.05 \mathrm{mM} 2$-mercaptoethanol -Sigma-Aldrich-, 10\% Rat T-STIM -Becton Dickinson, San Jose, CA, USA-) according to producer's specifications. MC/9 cells were cultured in suspension and maintained by the addition of fresh medium or its replacement. The cells were plated in 96well plates (Costar Corning Incorporate, NY, USA) at the density $6 \times 10^{4}$ cells/well for toluidine blue staining, in 24-well plates (Costar Corning Incorporate) at the density $5 \times 10^{5}$ cells/well for $\beta$ hexosaminidase release assay and viability studies.

\section{3. $O G D$}

Oxygen glucose deprivation (OGD) was performed in cortical neurons, MC/9 cells or co-cultures neurons-MC/9 as previously described (Lanzillotta et al., 2013). Briefly, cells were incubated with warm deoxygenated glucose-free balanced salt solution [(containing in $\mathrm{mM}$ ): $5.36 \mathrm{KCl}, 116.35 \mathrm{NaCl}, 0.81 \mathrm{MgSO}_{4}$ and 1.01 $\left.\mathrm{NaH}_{2} \mathrm{PO}_{4}\right)$ ] and transferred to an air-tight chamber fluxed with an anaerobic gas mixture $\left(95 \% \mathrm{~N}_{2}\right.$ and $\left.5 \% \mathrm{CO}_{2}\right)$ for $10 \mathrm{~min}$ to remove oxygen. The oxygen concentration was $<0.4 \%$ throughout the
OGD period, as assessed by an oxygen analyzer (Servomex $580 \mathrm{~A}$, Taylor Servomex, Edenbridge, UK). Cells were exposed to OGD at $37{ }^{\circ} \mathrm{C}$ for the indicated amount of time. Control cell cultures were incubated in a normal aerated incubator for the same time period. At the end of the OGD cells were allowed to recover in Neurobasal medium containing $0.4 \%$ B27 under normoxic conditions. In the experiments exploring MCs activation (pure MC/9 cells, or in cocultures with neurons), at the end of OGD, supplements (glucose, L-glutamine, penicillin/streptomycin and B27) were added directly to the glucose-free balanced salt solution in order to limit mastocytic activation derived from mechanical manipulation.

Cell lines were treated with PEA and luteolin, alone or in combination, before or after OGD, as described. The cell viability was estimated $24 \mathrm{~h}$ later for neurons and neurons-MC/9 co-cultures, or as specifically indicated for MC/9 cells. Mastocytic degranulation was assessed at the end of the OGD exposure.

\subsection{Interaction neurons-mast cells}

\subsubsection{Co-cultures neurons-MC/9 cells}

Cortical neuron cultures were plated in coated 24-well plates at 
the density $2.5 \times 10^{5}$ cells/well (Nunc-Thermo Scientific) as described above. MC/9 cells were plated in $6.5 \mathrm{~mm}$ diameter cell culture inserts (polyethylene terephthalate membrane, pore size $0.4 \mu \mathrm{m}$; Merck Millipore Billerica, MA, USA) at $10 \times 10^{3}$ cells per insert, and placed into the culture wells containing neurons following the manufacturer instructions (Fig. 2A).

\subsubsection{Preparation of MC/9-conditioned medium (CM)}

In a different experimental approach, neurons were incubated in $\mathrm{CM}$ derived from MC/9 cells subjected to OGD (Fig. 2D). MC/9 cells were plated at the density $7 \times 10^{5}$ cells/well in $60 \mathrm{~mm}$ diameter Petri dishes (Costar Corning Incorporate) and subjected to OGD as previously described. At the end of OGD period alone or at the end of OGD followed by incubation in recovery medium, supernatants were collected, samples were sedimented to remove $\mathrm{MC} / 9$ cells and particulates, and $500 \mu \mathrm{L}$ of $\mathrm{CM}$ were transferred to the neurons plated in 24-well plates (Nunc-Thermo Scientific).

\subsection{Degranulation assessment}

\subsubsection{Toluidine blue staining}

MCs abundant cytoplasmic granules can be purple stained using metachromatic dye such as toluidine blue. MC/9 cells were fixed with Immunofix (Bio-Optica, Milano, Italy) and stained with 0.005\% toluidine blue pH 2.5 (Merck, Darmstadt, Germany). The number of degranulated MCs was counted under a light microscope, considering degranulated the cells showing lack of cellular periphery integrity, pale purple staining or extrusion of granules out of the cells (Hu et al., 2005). The numbers of degranulated and total MCs were assessed from three randomly selected areas counting a minimum of 300 cells/field. The results were expressed as the degranulation percentage in each well.

\subsection{2. $\beta$-hexosaminidase release assay}

Degranulation was also evaluated measuring the exocytosis of $\beta$-hexosaminidase, an enzyme released from secretory granules of activated MCs. The protocol used is based on the method described by Kawahara (Kawahara, 2012). Briefly, $40 \mu \mathrm{L}$ of MCs supernatant was transferred to a 96-well microtiter plate (Greiner Bio-One, Frickenhausen, Germany) and incubated with $40 \mu \mathrm{L}$ of $1 \mathrm{mM}$ p-nitrophenyl-N-acetyl-d-glucosaminide (Sigma-Aldrich) in $0.1 \mathrm{M}$ citrate buffer $\left(\mathrm{pH} 4.5\right.$ ) for $1 \mathrm{~h}$ at $37^{\circ} \mathrm{C}$. The reaction was stopped by adding $160 \mu \mathrm{L}$ of $0.1 \mathrm{M}$ carbonate buffer ( $\mathrm{pH} 10.0)$. The total $\beta$-hexosaminidase value was estimated by lysing the cells with $0.1 \%$ Triton-X 100 . The absorbance of the mixture was measured at $405 \mathrm{~nm}$ using a microplate reader (iMark; Bio-Rad, Hercules, CA, USA). The relative value of the $\beta$-hexosaminidase activity was calculated as percentage of $\beta$-hexosaminidase released $/ \beta$ hexosaminidase total.

\subsection{Cytotoxicity assessment}

Neuronal and mastocytic injuries were evaluated by measuring the amount of lactate dehydrogenase (LDH) released into the culture medium using the CytoTox $96{ }^{\circledR}$ Non-Radioactive Cytotoxicity Assay (Promega Corporation, Wisconsin, USA) according to the manufacturer's instructions. In each well, LDH release was calculated relative to total releasable LDH obtained by incubating the cells for 30 min with $1 \%$ Triton X-100 at the end of each experiment. Values are expressed as a percentage of LDH released by cells exposed to OGD or CM over basal value.

\subsection{Statistical analysis}

All results were expressed as mean \pm SEM (standard error of the mean). Student's $t$-test and one-way ANOVA followed by
Dunnet's multiple comparison test were used for comparison of two or more than two groups, respectively. $P<0.05$ was considered statistically significant.

\section{Competing interests}

Mariella Fusco is an employee of Epitech Group s.r.l., the company that provided the molecules under investigation in this study. The other authors declare no conflict of interest.

\section{Authors' contributions}

EP designed the experiments, assessed cytotoxicity and mastocytic activation, analyzed the results and drafted the manuscript; VP participated in the study design and performed neurotoxicity experiments; RI assessed mastocytic degranulation; MB prepared neuronal cultures; $\mathrm{AL}$ and MM contributed to the data analysis; MF designed the experiments and participated in results interpretation; PT contributed to results interpretation; PFS contributed to experimental design and results interpretation; MP conceived of the study, coordinated the research, analyzed the results and drafted the manuscript.

\section{Acknowledgements}

This work was supported by H607962-1 ITN Neuroinflammation, Ricerca Finalizzata Ministero della Salute RF-2010-2315142, Fondazione CARIPLO 2014-0769.

\section{References}

Ahmad, A Genovese T Impellizzeri, D Crupi, R, Velardi, E, Marino, A, Esposito, E., Cuzzocrea, S., 2012. Reduction of ischemic brain injury by administration of palmitoylethanolamide after transient middle cerebral artery occlusion in rats. Brain Res, 1477, 45-58.

Attoub, S., Hassan, A.H., Vanhoecke, B., Iratni, R., Takahashi, T., Gaben, A.M., Bracke, M., Awad, S., John, A., Kamalboor, H.A., Al Sultan, M.A., Arafat, K., Gespach, C., Petroianu, G., 2011. Inhibition of cell survival, invasion, tumor growth and histone deacetylase activity by the dietary flavonoid luteolin in human epithelioid cancer cells. Eur. J. Pharmacol. 651, 18-25.

Barbierato, M., Facci, L., Marinelli, C., Zusso, M., Argentini, C., Skaper, S.D., Giusti, P. 2015. Co-ultramicronized palmitoylethanolamide/luteolin promotes the maturation of oligodendrocyte precursor cells. Sci. Rep. 5, 16676.

Caltagirone, C., Cisari, C., Schievano, C., Di Paola, R., Cordaro, M., Bruschetta, G., Esposito, E., Cuzzocrea, S., 2015. Co-ultramicronized palmitoylethanolamide luteolin in the treatment of cerebral ischemia: from rodent to man. Transl. Stroke Res.

Christy, A.L., Brown, M.A., 2007. The multitasking mast cell: positive and negative roles in the progression of autoimmunity. J. Immunol. 179, 2673-2679.

Cordaro, M., Impellizzeri, D., Paterniti, I., Bruschetta, G., Siracusa, R., De Stefano, D. Cuzzocrea, S., Esposito, E., 2015. Neuroprotective effects of Co-ultraPEALut on secondary inflammatory process and autophagy involved in traumatic brain injury. J. Neurotrauma 33 (1), 132-146.

Crupi, R., Paterniti, I., Ahmad, A., Campolo, M., Esposito, E., Cuzzocrea, S., 2013. Effects of palmitoylethanolamide and luteolin in an animal model of anxiety depression. CNS Neurol. Disord. Drug Targets 12, 989-1001.

D’Agostino, G., Russo, R., Avagliano, C., Cristiano, C., Meli, R., Calignano, A., 2012 Palmitoylethanolamide protects against the amyloid-beta25-35-induced learning and memory impairment in mice, an experimental model of Alzheimer disease. Neuropsychopharmacology 37, 1784-1792.

Dajas, F., Rivera-Megret, F., Blasina, F., Arredondo, F., Abin-Carriquiry, J.A., Costa, G. Echeverry, C., Lafon, L., Heizen, H., Ferreira, M., Morquio, A., 2003. Neuroprotection by flavonoids. Braz. J. Med. Biol. Res. 36, 1613-1620.

De Filippis, D., Luongo, L., Cipriano, M., Palazzo, E., Cinelli, M.P., de Novellis, V. Maione, S., Iuvone, T., 2011. Palmitoylethanolamide reduces granuloma-induced hyperalgesia by modulation of mast cell activation in rats. Mol. Pain 7, 3 .

De Filippis, D., Negro, L., Vaia, M., Cinelli, M.P., Iuvone, T., 2013. New insights in mast cell modulation by palmitoylethanolamide. CNS Neurol. Disord. Drug Targets 12, 78-83.

Dropp, J.J., 1979. Mast cells in the human brain. Acta Anat. 105, 505-513.

Esposito, E., Cordaro, M., Cuzzocrea, S., 2014. Roles of fatty acid ethanolamides (FAE) 
in traumatic and ischemic brain injury. Pharm. Res. 86, 26-31.

Esposito, E., Cuzzocrea, S., 2013. Palmitoylethanolamide in homeostatic and traumatic central nervous system injuries. CNS Neurol. Disord. Drug Targets 12, 55-61.

Esposito, E., Impellizzeri, D., Mazzon, E., Paterniti, I., Cuzzocrea, S., 2012. Neuroprotective activities of palmitoylethanolamide in an animal model of Parkinson's disease. PLoS One 7, e41880.

Esposito, E., Paterniti, I., Mazzon, E., Genovese, T., Di Paola, R., Galuppo, M., Cuzzocrea, S., 2011. Effects of palmitoylethanolamide on release of mast cell peptidases and neurotrophic factors after spinal cord injury. Brain Behav. Immun. 25, 1099-1112.

Facci, L., Dal Toso, R., Romanello, S., Buriani, A., Skaper, S.D., Leon, A., 1995. Mast cells express a peripheral cannabinoid receptor with differential sensitivity to anandamide and palmitoylethanolamide. Proc. Natl. Acad. Sci. USA 92, 3376-3380.

Galli, S.J., Nakae, S., Tsai, M., 2005. Mast cells in the development of adaptive immune responses. Nat. Immunol. 6, 135-142.

Garg, P., Duncan, R.S., Kaja, S., Koulen, P., 2010. Intracellular mechanisms of $\mathrm{N}$-acylethanolamine-mediated neuroprotection in a rat model of stroke. Neuroscience 166, 252-262.

Guerra-Araiza, C., Alvarez-Mejia, A.L., Sanchez-Torres, S., Farfan-Garcia, E., Mondragon-Lozano, R., Pinto-Almazan, R., Salgado-Ceballos, H., 2013. Effect of natural exogenous antioxidants on aging and on neurodegenerative diseases. Free Radic. Res. 47, 451-462.

Hansen, H.S., 2010. Palmitoylethanolamide and other anandamide congeners. Proposed role in the diseased brain. Exp. Neurol. 224, 48-55.

Henderson, W.R., Chi, E.Y., Klebanoff, S.J., 1980. Eosinophil peroxidase-induced mast cell secretion. J. Exp. Med. 152, 265-279.

Hu, W., Xu, L., Pan, J., Zheng, X., Chen, Z., 2004. Effect of cerebral ischemia on brain mast cells in rats. Brain Res. 1019, 275-280.

Hu, W., Shen, Y., Fu, Q., Dai, H., Tu, H., Wei, E., Luo, J., Chen, Z., 2005. Effect of oxygen-glucose deprivation on degranulation and histamine release of mast cells. Cell Tissue Res. 322, 437-441.

Impellizzeri, D., Esposito, E., Di Paola, R., Ahmad, A., Campolo, M., Peli, A., Morittu, V.M., Britti, D., Cuzzocrea, S., 2013. Palmitoylethanolamide and luteolin ameliorate development of arthritis caused by injection of collagen type II in mice. Arthritis Res Ther. 15, R192.

Impellizzeri, D., Ahmad, A., Bruschetta, G., Di Paola, R., Crupi, R., Paterniti, I., Esposito, E., Cuzzocrea, S., 2015. The anti-inflammatory effects of palmitoylethanolamide (PEA) on endotoxin-induced uveitis in rats. Eur. J. Pharmacol 761, 28-35.

Jeon, I.H., Kim, H.S., Kang, H.J., Lee, H.S., Jeong, S.I., Kim, S.J., Jang, S.I., 2014. Antiinflammatory and antipruritic effects of luteolin from Perilla (P. frutescens L.) leaves. Molecules 19, 6941-6951.

Jin, M., Son, K.H., Chang, H.W., 2011. Luteolin-7-O-glucoside suppresses leukotriene $\mathrm{C}(4)$ production and degranulation by inhibiting the phosphorylation of mitogen activated protein kinases and phospholipase Cgamma1 in activated mouse bone marrow-derived mast cells. Biol. Pharm. Bull. 34, 1032-1036.

Kawahara, T., 2012. Establishment and characterization of mouse bone marrowderived mast cell hybridomas. Exp. Cell Res. 318, 2385-2396.

Kritas, S.K., Saggini, A., Varvara, G., Murmura, G., Caraffa, A., Antinolfi, P., Toniato, E., Pantalone, A., Neri, G., Frydas, S., Rosati, M., Tei, M., Speziali, A., Saggini, R., Pandolfi, F., Cerulli, G., Theoharides, T.C., Conti, P., 2013. Luteolin inhibits mast cell-mediated allergic inflammation. J. Biol. Regul. Homeost. Agents 27, 955-959.

Lanzillotta, A., Pignataro, G., Branca, C., Cuomo, O., Sarnico, I., Benarese, M., Annunziato, L., Spano, P., Pizzi, M., 2013. Targeted acetylation of NF-kappaB/RelA and histones by epigenetic drugs reduces post-ischemic brain injury in mice with an extended therapeutic window. Neurobiol. Dis. 49C, 177-189.

Lanzillotta, A., Sarnico, I., Ingrassia, R., Boroni, F., Branca, C., Benarese, M., Faraco, G. Blasi, F., Chiarugi, A., Spano, P., Pizzi, M., 2010. The acetylation of RelA in Lys310 dictates the NF-kappaB-dependent response in post-ischemic injury. Cell Death Dis. 1, e96.

Lin, Y., Shi, R., Wang, X., Shen, H.M., 2008. Luteolin, a flavonoid with potential for cancer prevention and therapy. Curr. Cancer Drug Targets 8 (7), 634-646.

Lindsberg, P.J., Strbian, D., Karjalainen-Lindsberg, M.L., 2010. Mast cells as early responders in the regulation of acute blood-brain barrier changes after cerebral ischemia and hemorrhage. J. Cereb. Blood Flow. Metab. 30, 689-702.

Lopez-Lazaro, M., 2009. Distribution and biological activities of the flavonoid luteolin. Mini Rev. Med. Chem. 9, 31-59.

LoVerme, J., Russo, R., La Rana, G., Fu, J., Farthing, J., Mattace-Raso, G., Meli, R., Hohmann, A., Calignano, A., Piomelli, D., 2006. Rapid broad-spectrum analgesia through activation of peroxisome proliferator-activated receptor-alpha. J. Pharm. Exp. Ther. 319, 1051-1061.

Marshall, J.S., 2004. Mast-cell responses to pathogens. Nat. Rev. Immunol. 4, 787-799.

Masini, E., Palmerani, B., Gambassi, F., Pistelli, A., Giannella, E., Occupati, B., Ciuffi, M., Sacchi, T.B., Mannaioni, P.F., 1990. Histamine release from rat mast cells induced by metabolic activation of polyunsaturated fatty acids into free radicals. Biochem Pharmacol. 39, 879-889.

Mattila, O.S., Strbian, D., Saksi, J., Pikkarainen, T.O., Rantanen, V., Tatlisumak, T., Lindsberg, P.J., 2011. Cerebral mast cells mediate blood-brain barrier disruption in acute experimental ischemic stroke through perivascular gelatinase activation. Stroke 42, 3600-3605.

Metz, M., Grimbaldeston, M.A., Nakae, S., Piliponsky, A.M., Tsai, M., Galli, S.J., 2007. Mast cells in the promotion and limitation of chronic inflammation. Immunol. Rev. 217, 304-328.

Nelissen, S., Lemmens, E., Geurts, N., Kramer, P., Maurer, M., Hendriks, J., Hendrix, S., 2013. The role of mast cells in neuroinflammation. Acta Neuropathol. 125, 637-650.

Paterniti, I., Di Paola, R., Campolo, M., Siracusa, R., Cordaro, M., Bruschetta, G., Tremolada, G., Maestroni, A., Bandello, F., Esposito, E., Zerbini, G., Cuzzocrea, S., 2015. Palmitoylethanolamide treatment reduces retinal inflammation in streptozotocin-induced diabetic rats. Eur. J. Pharmacol. 769, 313-323.

Paterniti, I., Cordaro, M., Campolo, M., Siracusa, R., Cornelius, C., Navarra, M., Cuzzocrea, S., Esposito, E., 2014. Neuroprotection by association of palmitoylethanolamide with luteolin in experimental alzheimer's disease models: the control of neuroinflammation. CNS Neurol. Disord. Drug Targets.

Paterniti, I., Impellizzeri, D., Di Paola, R., Navarra, M., Cuzzocrea, S., Esposito, E., 2013. A new co-ultramicronized composite including palmitoylethanolamide and luteolin to prevent neuroinflammation in spinal cord injury. J. Neuroinflamm. 10, 91.

Qiao, H., Dong, L., Zhang, X., Zhu, C., Wang, L., Liu, Z., Chen, L., Xing, Y., Wang, C., Li, Y., 2012. Protective effect of luteolin in experimental ischemic stroke: upregulated SOD1, CAT, Bcl-2 and claudin-5, down-regulated MDA and Bax expression. Neurochem. Res. 37, 2014-2024.

Qiao, H., Zhang, X., Zhu, C., Dong, L., Wang, L., Xing, Y., Wang, C., Ji, Y., Cao, X., 2014. Luteolin downregulates TLR4, TLR5, NF-kappaB and p-p38MAPK expression, upregulates the p-ERK expression, and protects rat brains against focal ischemia. Brain Res. 1448, 71-81.

Sarnico, I., Lanzillotta, A., Boroni, F., Benarese, M., Alghisi, M., Schwaninger, M., Inta, I., Battistin, L., Spano, P., Pizzi, M., 2009. NF-kappaB p50/RelA and c-Rel-containing dimers: opposite regulators of neuron vulnerability to ischaemia. J. Neurochem. 108, 475-485.

Scuderi, C., Stecca, C., Valenza, M., Ratano, P., Bronzuoli, M.R., Bartoli, S., Steardo, L., Pompili, E., Fumagalli, L., Campolongo, P., Steardo, L., 2014. Palmitoylethanolamide controls reactive gliosis and exerts neuroprotective functions in a rat model of Alzheimer's disease. Cell Death Dis. 5, e1419.

Scuderi, C., Valenza, M., Stecca, C., Esposito, G., Carratu, M.R., Steardo, L., 2012. Palmitoylethanolamide exerts neuroprotective effects in mixed neuroglial cultures and organotypic hippocampal slices via peroxisome proliferator-activated receptor-alpha. J. Neuroinflamm. 9, 49.

Silver, R., Curley, J.P., 2013. Mast cells on the mind: new insights and opportunities. Trends Neurosci. 36, 513-521.

Silverman, A.J., Sutherland, A.K., Wilhelm, M., Silver, R., 2000. Mast cells migrate from blood to brain. J. Neurosci. 20, 401-408.

Siracusa, R., Paterniti, I., Impellizzeri, D., Cordaro, M., Crupi, R., Navarra, M., Cuzzocrea, S., Esposito, E., 2015. The association of palmitoylethanolamide with luteolin decreases neuroinflammation and stimulates autophagy in Parkinson's disease model. CNS Neurol. Disord. Drug Targets.

Skaper, S.D. Facci, L. Fusco, M., Della Valle, M.F., Zusso, M., Costa, B., Giusti, P., 2014. Palmitoylethanolamide, a naturally occurring disease-modifying agent in neuropathic pain. Inflammopharmacology 22, 79-94.

Skaper, S.D., Facci, L., Giusti, P., 2013. Glia and mast cells as targets for palmitoylethanolamide, an anti-inflammatory and neuroprotective lipid mediator. Mol. Neurobiol.

Skaper, S.D., Buriani, A., Dal Toso, R., Petrelli, L., Romanello, S., Facci, L., Leon, A., 1996a. The ALIAmide palmitoylethanolamide and cannabinoids, but not anandamide, are protective in a delayed postglutamate paradigm of excitotoxic death in cerebellar granule neurons. Proc. Natl. Acad. Sci. USA 93, 3984-3989.

Skaper, S.D., Facci, L., Romanello, S., Leon, A., 1996b. Mast cell activation causes delayed neurodegeneration in mixed hippocampal cultures via the nitric oxide pathway. J. Neurochem. 66, 1157-1166.

Strbian, D., Karjalainen-Lindsberg, M.L., Tatlisumak, T., Lindsberg, P.J., 2006. Cerebral mast cells regulate early ischemic brain swelling and neutrophil accumulation. J. Cereb. Blood Flow. Metab. 26, 605-612.

Strbian, D., Kovanen, P.T., Karjalainen-Lindsberg, M.L., Tatlisumak, T., Lindsberg, P.J., 2009. An emerging role of mast cells in cerebral ischemia and hemorrhage. Ann. Med. 41, 438-450.

Taliou, A., Zintzaras, E., Lykouras, L., Francis, K., 2013. An open-label pilot study of a formulation containing the anti-inflammatory flavonoid luteolin and its effects on behavior in children with autism spectrum disorders. Clin. Ther. 35, 592-602.

Theoharides, T.C., Asadi, S., Panagiotidou, S., 2012. A case series of a luteolin formulation (NeuroProtek $(R)$ ) in children with autism spectrum disorders. Int. J. Immunopathol. Pharmacol. 25, 317-323.

Theoharides, T.C., Asadi, S., Patel, A.B., 2013. Focal brain inflammation and autism. J. Neuroinflamm. 10, 46.

Theoharides, T.C., 1990. Mast cells: the immune gate to the brain. Life Sci. 46, 607-617.

Theoharides, T.C., 2009. Luteolin as a therapeutic option for multiple sclerosis. J. Neuroinflamm. 6, 29.

Zhao, G., Zang, S.Y., Jiang, Z.H., Chen, Y.Y., Ji, X.H., Lu, B.F., Wu, J.H., Qin, G.W., Guo, L. H., 2011. Postischemic administration of liposome-encapsulated luteolin prevents against ischemia-reperfusion injury in a rat middle cerebral artery occlusion model. J. Nutr. Biochem. 22, 929-936. 